\title{
Potential therapeutic effects of $N$ - butylidenephthalide from Radix Angelica Sinensis (Danggui) in human bladder cancer cells
}

Sheng-Chun Chiu ${ }^{1,2,3 \dagger}$, Tsung-Lang Chiu ${ }^{4 \dagger}$, Sung-Ying Huang ${ }^{5}$, Shu-Fang Chang ${ }^{1}$, Shee-Ping Chen ${ }^{6}$, Cheng-Yoong Pang ${ }^{7,8^{*}}$ and Teng-Fu Hsieh ${ }^{9,10^{*}}$

\begin{abstract}
Background: N-butylidenephthalide (BP) isolated from Radix Angelica Sinensis (Danggui) exhibits anti-tumorigenic effect in various cancer cells both in vivo and in vitro. The effect of BP in bladder cancer treatment is still unclear and worth for further investigate.

Methods: Changes of patients with bladder cancer after Angelica Sinensis exposure were evaluated by analysis of Taiwan's National Health Insurance Research Database (NHIRD) database. The anti-proliferative effect of BP on human bladder cancer cells was investigated and their cell cycle profiles after BP treatment were determined by flow cytometry. BP-induced apoptosis was demonstrated by Annexin V-FITC staining and TUNEL assay, while the expressions of apoptosis-related proteins were determined by western blot. The migration inhibitory effect of BP on human bladder cancer cells were shown by trans-well and wound healing assays. Tumor model in NOD-SCID mice were induced by injection of BFTC human bladder cancer cells.

Results: The correlation of taking Angelica sinensis and the incidence of bladder cancer in NHIRD imply that this herbal product is worth for further investigation. BP caused bladder cancer cell death in a time- and dose- dependent manner and induced apoptosis via the activation of caspase-9 and caspase-3. BP also suppressed the migration of bladder cancer cells as revealed by the trans-well and wound healing assays. Up-regulation of E-cadherin and down-regulation of N-cadherin were evidenced by real-time RT-PCR analysis after BP treatment in vitro. Besides, in combination with BP, the sensitivity of these bladder cancer cells to cisplatin increased significantly. BP also suppressed BFTC xenograft tumor growth, and caused $44.2 \%$ reduction of tumor volume after treatment for 26 days.

Conclusions: BP caused bladder cancer cell death through activation of mitochondria-intrinsic pathway. BP also suppressed the migration and invasion of these cells, probably by modulating EMT-related genes. Furthermore, combination therapy of BP with a lower dose of cisplatin significantly inhibited the growth of these bladder cancer cell lines. The incidence of bladder cancer decreased in patients who were exposed to Angelica sinensis, suggesting that BP could serve as a potential adjuvant in bladder cancer therapy regimen.
\end{abstract}

Keywords: Apoptosis, Bladder cancer, Combination therapy, Metastasis, n-butylidenephthalide, NHIRD

\footnotetext{
*Correspondence: cypang@mail.tcu.edu.tw; pchdf95@gmail.com

${ }^{\dagger}$ Equal contributors

${ }^{7}$ Department of Medical Research, Hualien Tzu Chi Hospital, Buddhist Tzu Chi

Medical Foundation, Hualien, Taiwan

${ }^{9}$ Department of Urology, Taichung Tzu Chi Hospital, Buddhist Tzu Chi

Medical Foundation, Section 1, Fengxing Road, Tanzi Dist., Taichung City 427,

Taiwan

Full list of author information is available at the end of the article
} 


\section{Background}

Bladder cancer is one of the common cancer in the United States and many other parts of the world $[1,2]$. The estimated numbers of new cases are expected to reach 74,000/year in 2015 in the United States [2]. Previous studies showed that approximately $30 \%$ of newly diagnosed superficial bladder cancers were multifocal, however, $60-70 \%$ of these superficial bladder cancers recurred, and $10-20 \%$ of them would undergo stage progression to a muscle-invasive or metastatic disease [3]. The effect of chemotherapy or other systemic treatment for bladder cancer is limited [4]. Although Platinum-based chemotherapy is commonly used for bladder cancer $[5,6]$, radical cystectomy and systemic chemotherapy are suggested for invasive bladder cancer. These treatment regimens usually fail in $95 \%$ patients: at least half of the invasive bladder cancer patients still die of metastases within 2 years after diagnosis, and with less than 10\% 5-year survival rate $[2,7]$.

Several nutrients and non-nutritive phytochemicals have been evaluated in interventional trials for their potential as cancer chemo-preventive agents. Nontraditional treatments using herbs and dietary supplements have also been considered as alternative therapy for cancer. Angelica sinensis (Oliv.) Diels (Umbelliferae), which is pronounced as "Danggui" in Mandarin, is one of the most commonly used herbs in traditional Chinese medicine (TCM). It is clinically administrated to replenish blood and to treat several gynecological symptoms such as menstrual disorders in women. $N$-butylidenephthalide (BP), an active compound isolated from the chloroform extract of Angelica sinensis, has been identified as having growth-inhibitory and apoptosis-induction effects on various cancer cells [8-13]. These findings highlight the potential therapeutic role of $\mathrm{BP}$ in clinical application. However, the effect of BP on human bladder cancer cells is still unclear and worth further investigation.

Our aim in this study was to investigate the probable anti-proliferative effect of BP on bladder cancer cells, and to determine the signaling pathway that might involve. In addition, NOD-SCID mice xenograft tumor model was used to evaluate the antitumor effect of BP on bladder cancer in vivo. On the other hand, since Taiwan's National Health Insurance Research Database (NHIRD) has been successfully used in epidemiological studies of cancer and Chinese herbal products (CHPs) $[14,15]$, we also investigated the correlation of taking Angelica sinensis and the incidence of bladder cancer in Taiwan.

\section{Methods}

Cell proliferation assay, western blot and cell cycle analysis were performed as previously described [9], with further details were provided in Additional file 1. For
RNA isolation, cell migration and invasion assay, and quantitative RT-PCR, see Additional file 1.

\section{Cell culture}

Human bladder cancer cell line TCCSUP was purchased from ATCC (American Type Culture Collection, Manassas, VA). Human bladder cancer cell lines 5637, T24, and BFTC (BFTC 905) were purchased from BCRC (Bioresource Collection and Research Center, Hsinchu, Taiwan). Cells were cultured in appropriate culture medium and supplements according to the suggestion of ATCC and BCRC, respectively. Cell lines were authenticated annually by shorttandem repeat analysis and routinely tested for mycoplasma contamination (BCRC).

\section{Chemicals and antibodies}

BP $\left(\mathrm{C}_{12} \mathrm{H}_{12} \mathrm{O}_{2}, 95 \%\right)$ was purchased from Lancaster Synthesis Ltd. (Newgate Morecambe, UK). Cisplatin, dimethyl sulfoxide (DMSO), [3-(4,5-dimethyl thizol-2-yl)-2,5-diphenyl tetrazolium bromide] (MTT), crystal violet, DSD, Tween-20, methanol, and horseradish peroxidaseconjugated secondary antibodies were purchased from Sigma Chemical Co. (St. Louis, MO, USA). The primary antibodies were all purchased from Cell Signaling Technology, Inc., (Danvers, MA, USA). Polyvinyldifluoride (PVDF) membranes, BSA protein assay kit and chemiluminescence reagents were purchased from Amersham Biosciences (Arlington Heights, IL, USA).

\section{TUNEL assay}

Human bladder cancer cells were cultured in the presence or absence of BP $(60 \mu \mathrm{g} / \mathrm{ml})$ for $72 \mathrm{~h}$ and then examined for apoptosis with TUNEL assay (In Situ Cell Death Detection Kit, Roche) according to the manufacturer's instructions.

\section{Annexin V-FITC staining}

Human bladder cancer cells were cultured in the presence or absence of BP $(60 \mu \mathrm{g} / \mathrm{ml})$ for 3,18 and $24 \mathrm{~h}$, as indicated. The vehicle control group was treated with 0.2\% DMSO only. Apoptotic cell death was examined using annexin V-FITC detection kits according to the manufacturer's instructions (BD Biosciences, San Diego, CA, USA). Ten thousand events were acquired for each sample and analyzed by Accuri C6 flow cytometer with CFlow ${ }^{\oplus}$ software.

\section{Animal studies}

Tumors were generated as previously described [9] and further details were provided in Additional file 1.

\section{Patients and study design}

Taiwan implemented a National Health Insurance (NHI) program in 1995 to provide comprehensive health care 
coverage. Enrollment in this government-run, universal, single-payer insurance system is mandatory and up to 99\% of the 23 million residents of Taiwan receive medical care through the NHI program. In addition, $>97 \%$ of the hospitals and clinics in Taiwan are contracted to provide health care services under the NHI [16]. All data related to these services are collected and input into the NHIRD by the National Health Research Institute to provide a comprehensive record of medical care. The data consist of ambulatory care records, in-patient care records, and registration files of the insured, and the database includes all claims data from the NHI program. The NHI Bureau randomly reviews the charts of one out of every 100 ambulatory cases and one out of every 20 in-patient cases, as well as conduct patient interviews to verify the accuracy of the diagnosis [17].

This study used the NHIRD collected within 2003 to 2009. The study design featured a study cohort and a comparison cohort. Patients with newly-diagnosed with bladder cancer (International Classification of Diseases, Ninth Revision, Clinical Modification (ICD-9-CM) code 188.XX) before the index date were excluded. Patients who received CHPs containing Angelica sinensis between 2002 and 2009 were then identified as the study cohort (the exposure group). The date of initiation of Angelica sinensis exposure was used as the patient's index date. The control cohort (the non-exposure group) included all of the other patients without taking CHPs therapy, and propensity score matching with age and gender was applied to select the controls.

\section{Charlson Comorbidity Index Score}

The Charlson Comorbidity Index Score (CCIS) is a widely accepted measure for risk adjustment in administrative claims data sets [18-20]. The CCIS were calculated for each patient by assigning 1 point each for myocardial infarct, congestive heart failure, peripheral vascular disease, dementia, cerebrovascular disease, chronic lung disease, connective tissue disease, ulcer, chronic liver disease and diabetes by assigning 2 points each for hemiplegia, moderate or severe kidney disease, diabetes with end organ damage, tumor, leukemia and lymphoma, 3 points for moderate or severe liver disease and 6 points each for malignant tumor, metastasis and acquired immune deficiency syndrome.

\section{Other variables}

The study subjects were classified into three groups: (1) low SES, <583 US \$ per month (New Taiwan Dollars [NTD] 17,500); (2) moderate SES, 583-833 US \$ per month (NTD 17500-25,000); and (3) high SES, $\geq 833$ US $\$$ per month ( $\geq$ NTD 25001) [21]. Low income was set at NTD 17500 because this was the governmentstipulated minimum wage for full-time employees in
Taiwan in 2009. The geographic regions where the individuals resided were recorded as northern, central, southern, and eastern Taiwan.

\section{Statistical analysis}

All data were shown as mean \pm S.D. Statistical differences were analyzed using the Student's t-test for normally distributed values and by nonparametric Mann-Whitney $U$-test for values with a non-normal distribution. Significant differences between groups were evaluated using analysis of variance (ANOVA) with Games-Howell test as post-hoc test. Pearson's chi-square test was used for categorical variables such as gender, SES, geographic region of residence, co-morbidities and incidence of bladder cancer.

\section{Results}

The incidence of bladder cancer with or without Angelica sinensis exposure in NHIRD database

From 2002 to 2009, NHIRD data from 17,091 patients who had taken Angelica sinensis (solely or as one of the ingredients) were included for analysis. Table 1 shows the number of patients, age, gender, and distribution of patients in different comorbidities. The mean age at diagnosis was $53.8 \pm 10.9$ years. Severe comorbidity (CCIS $\geqq 4$ ) was noted in $4.5 \%$ patients who were exposed to Angelica sinensis. At the end of the follow-up period, 30 patients had bladder cancer, including $8(0.05 \%)$ in the exposure group, and $22(0.13 \%)$ in the control group (Table $2 ; p=$ 0.0106 , Relative risk $=0.38$ ). The result prompted us to investigate the physiological effect of BP, one of the major component found in the chloroform extract fraction of Angelica sinensis, on human bladder cancer cells.

\section{BP inhibited proliferation of human bladder cancer cells}

To determine the cytotoxicity effect of BP on bladder cancer cells, 4 human bladder cancer cell lines (5637, BFTC, T24, and TCCSUP) were treated with increasing concentration (12.5 to $100 \mu \mathrm{g} / \mathrm{ml})$ of BP for 24 and $48 \mathrm{~h}$, respectively, and subsequently evaluated for cell viability using the MTT assay. As shown in Fig. 1a, all 4 bladder cancer cell lines showed rounding up and shrinkage of cells as compared to the control cells after $24 \mathrm{~h}$ of BP treatment. We further demonstrated that BP significantly reduced the viability of 4 bladder cancer cell lines in a dose- and time-dependent manner by MTT assay (Fig. 1b). Treatment of 4 bladder cancer cells with 50 or $75 \mu \mathrm{g} / \mathrm{ml}$ BP for $48 \mathrm{~h}$ resulted in $56.3 \%$ and $42 \%$ (5637), $63.7 \%$ and $57.4 \%$ (BFTC), $63.5 \%$ and $39.4 \%$ (T24), $51.9 \%$ and $21.6 \%$ (TCCSUP) cell survival, respectively. The $\mathrm{IC}_{50}$ at $48 \mathrm{~h}$ of BP treatment in bladder cancer cells were: $5637,67.1 \mu \mathrm{g} / \mathrm{ml}$; BFTC, $64.1 \mu \mathrm{g} / \mathrm{ml}$; T24, $59.9 \mu \mathrm{g} /$ $\mathrm{ml}$; TCCSUP, $53 \mu \mathrm{g} / \mathrm{ml}$, respectively. Thus, we used $60 \mu \mathrm{g} / \mathrm{ml} \mathrm{BP}$ in the subsequent experiments. 
Table 1 Characteristics of the patients with or without Angelica sinensis exposure from 2002 to 2009 in Taiwan $(n=34,182)$

\begin{tabular}{|c|c|c|c|}
\hline Variables & $\begin{array}{l}\text { Non-exposure } \\
\mathrm{N}(\%)\end{array}$ & $\begin{array}{l}\text { Exposure } \\
\mathrm{N}(\%)\end{array}$ & $p$-value \\
\hline Total & $17,091(50.0)$ & $17,091(50.0)$ & \\
\hline Mean age,years $( \pm S D)$ & $53.8 \pm 10.9$ & $53.8 \pm 10.9$ & 1.00 \\
\hline Sex & & & 1.00 \\
\hline Male & $6244(36.5)$ & $6244(36.5)$ & \\
\hline Female & $10,847(63.5)$ & $10,847(63.5)$ & \\
\hline CCIS score & & & $<.0001$ \\
\hline Mean \pm SD & $0.40 \pm 1.00$ & $0.76 \pm 1.43$ & \\
\hline $0-1$ & $15,523(90.8)$ & $14,174(82.9)$ & \\
\hline $2-3$ & $1259(7.4)$ & $5157(12.6)$ & \\
\hline Over 4 & $309(1.8)$ & $760(4.5)$ & \\
\hline \multicolumn{4}{|l|}{ Comborbidities } \\
\hline Diabetes mellitus & $1181(6.9)$ & $1590(9.3)$ & $<.0001$ \\
\hline Socioeconomic status & & & $<.0001$ \\
\hline Low SES $(<=17,500)$ & $7510(43.9)$ & $6954(40.6)$ & \\
\hline ModerateSES $(17,500 \sim 25,000)$ & $5979(35.0)$ & $6249(36.6)$ & \\
\hline High SES(> = 25,000) & $3602(21.1)$ & $3897(22.8)$ & \\
\hline Urbanization & & & $<.0001$ \\
\hline Urban & $5428(31.8)$ & $5130(30.0)$ & \\
\hline Suburban & $7390(43.2)$ & $7872(46.1)$ & \\
\hline Rural & $4273(25.0)$ & $4089(23.9)$ & \\
\hline Geographic region & & & $<.0001$ \\
\hline Northern & $9090(53.2)$ & $6672(39.0)$ & \\
\hline Central & $2204(12.9)$ & $4728(27.7)$ & \\
\hline Southern & $5335(31.2)$ & $5319(31.1)$ & \\
\hline Eastern & $462(2.7)$ & $372(2.2)$ & \\
\hline
\end{tabular}

\section{BP induced sub-G1 accumulation in human bladder cancer} cells

To investigate the role of apoptosis in BP-induced bladder cancer cell death, annexin V-FITC staining and flow cytometric analysis was performed (Fig. 2). Human bladder cancer cells treated with $60 \mu \mathrm{g} / \mathrm{ml} \mathrm{BP}$ for 0 to $24 \mathrm{~h}$ were analyzed by flow cytometry (Fig. 2a). The annexin V-FITC positive and PI negative populations (Q3-LR) were increased after BP treatment as compared to the control group. Apoptosis was noted as early at $3 \mathrm{~h}$ after BP treatment in 4 bladder cancer cell lines. After BP treatment for $24 \mathrm{~h}$, the Q3-LR portion increased to $12.6 \%$ (5637), 34.8\% (BFTC), 2.2\% (T24) and 23.6\% (TCCSUP), respectively

Table 2 Bladder cancer incidence and relative risk

\begin{tabular}{llll}
\hline Characteristics & $\begin{array}{l}\text { Bladder cancer } \\
\text { N }(\%)\end{array}$ & Relative risk & $p$-value \\
\hline Non-exposure to Angelica sinensis & $22(0.13 \%)$ & 1 & 0.01 \\
Exposure to Angelica sinensis & $8(0.05 \%)$ & 0.38 & \\
\hline
\end{tabular}

(Fig. 2b). The Q3-LR portion of T24 was significantly lower than other bladder cancer cell lines after BP treatment and worth for further clarification. The appearance of Sub-G1 cell population can be evaluated as the degree of apoptotic cell death. As showed in Fig. 2c, treatment with $60 \mu \mathrm{g} / \mathrm{ml} \mathrm{BP}$ induced the accumulation of sub-G1 portion in 4 bladder cancer cell lines. After BP treatment for $48 \mathrm{~h}$, the sub-G1 portion increased to $37.6 \%$ (5637), 12.4\% (BFTC), 23.4\% (T24) and 19.7\% (TCCSUP), respectively, in 4 bladder cancer cell lines (Fig. 2d). Together, these data suggested that apoptosis induction was involved in BP-induced bladder cancer cell death.

\section{BP induced mitochondrial-mediated apoptosis in human bladder cancer cells}

TUNEL assay was performed to detect apoptotic cells that undergo DNA degradation during the late stage of apoptosis. Cells treated with $60 \mu \mathrm{g} / \mathrm{ml}$ BP for $72 \mathrm{~h}$ were evaluated by TUNEL assay. The TUNEL positive cells (green fluorescence) were significantly increased after BP treatment as compared to the control (Fig. 3a). Activation of caspase proteins is the crucial steps for apoptosis induction. The key cysteine proteases, caspase -9 and -3 , play critical roles in the activation of mitochondria-mediated apoptosis. The involvement of these caspases in BPinduced apoptosis was investigated in these bladder cancer cell lines. Cleavages of caspase -9 and -3 increased in bladder cancer cells from 0 to $48 \mathrm{~h}$ after BP treatment (Fig. $3 \mathrm{~b})$. These data suggested that BP induced mitochondriamediated apoptosis in bladder cancer cells. To examine the pivotal role of caspase 3 in BP-induced apoptosis, cells were pretreated with Z-DEVD-fmk (caspase 3 inhibitor, $20 \mu \mathrm{M}$ ) for $1 \mathrm{~h}$, and then treated with $60 \mu \mathrm{g} / \mathrm{ml} \mathrm{BP}$ for $48 \mathrm{~h}$ (Fig. 3c). The pretreatment of inhibitor partly blocked BP-induced cell death $(63 \%$ in $5637,66.3 \%$ in BFTC, $73.6 \%$ in T24, and $62.5 \%$ in TCCSUP, respectively) as compared to the BP alone group (49.9\% in 5637, 53.4\% in BFTC, $62.6 \%$ in T24, and 50.2\% in TCCSUP, respectively). Although to a lesser extent, these data further confirmed that caspasedependent pathway was involved in BP-induced human bladder cancer cell death.

\section{BP suppressed human bladder cancer cells migration and invasion in vitro}

The inhibitory effect of BP in bladder cancer cells were further examined by wound closure assay (Fig. 4). The control cells migrated and filled the scratched area within $24 \mathrm{~h}$. The cell-free area filled by migrated cells were quantified and compared. As shown in Fig. 4, these results demonstrated that the migration of BP-treated bladder cancer cells was inhibited: the extents of inhibition of migration by $60 \mu \mathrm{g} / \mathrm{ml} \mathrm{BP}$ at $24 \mathrm{~h}$ for 5637 , BFTC, T24 and TCCSUP were $72 \%, 94.5 \%, 53.6 \%$ and $100.4 \%$, respectively. These data suggested that BP 




inhibited the migration of human bladder cancer cells in a time- and dose-dependent manner.

The inhibitory effect of BP on bladder cancer cell was further explored with trans-well migration and invasion assay. The migration of BP-treated $(60 \mu \mathrm{g} / \mathrm{ml})$ human bladder cancer cells decreased significantly as compared to the non-treated cells (Fig. 5a). The migrated cells accounted for $66.9 \%$ (5637), 59.6\% (BFTC), 63.2\% (T24) and $36.1 \%$ (TCCSUP), respectively, as compared to the non-treated cells. Similar results were obtained in the invasion assay, the migrated cells accounted for 39\% (5637), $72.9 \%$ (BFTC), $54.5 \% \quad$ (T24) and $11.1 \%$ (TCCSUP), respectively, as compared to the control cells. Taken together, we showed that BP suppressed cell migration and invasion of human bladder cancer cells.

\section{Modulation of epithelial-mesenchymal transition by BP}

Modulation of genes that are involved in epithelialmesenchymal transition (EMT) has been regarded as a hallmark of cancer metastasis. To evaluate whether BP 

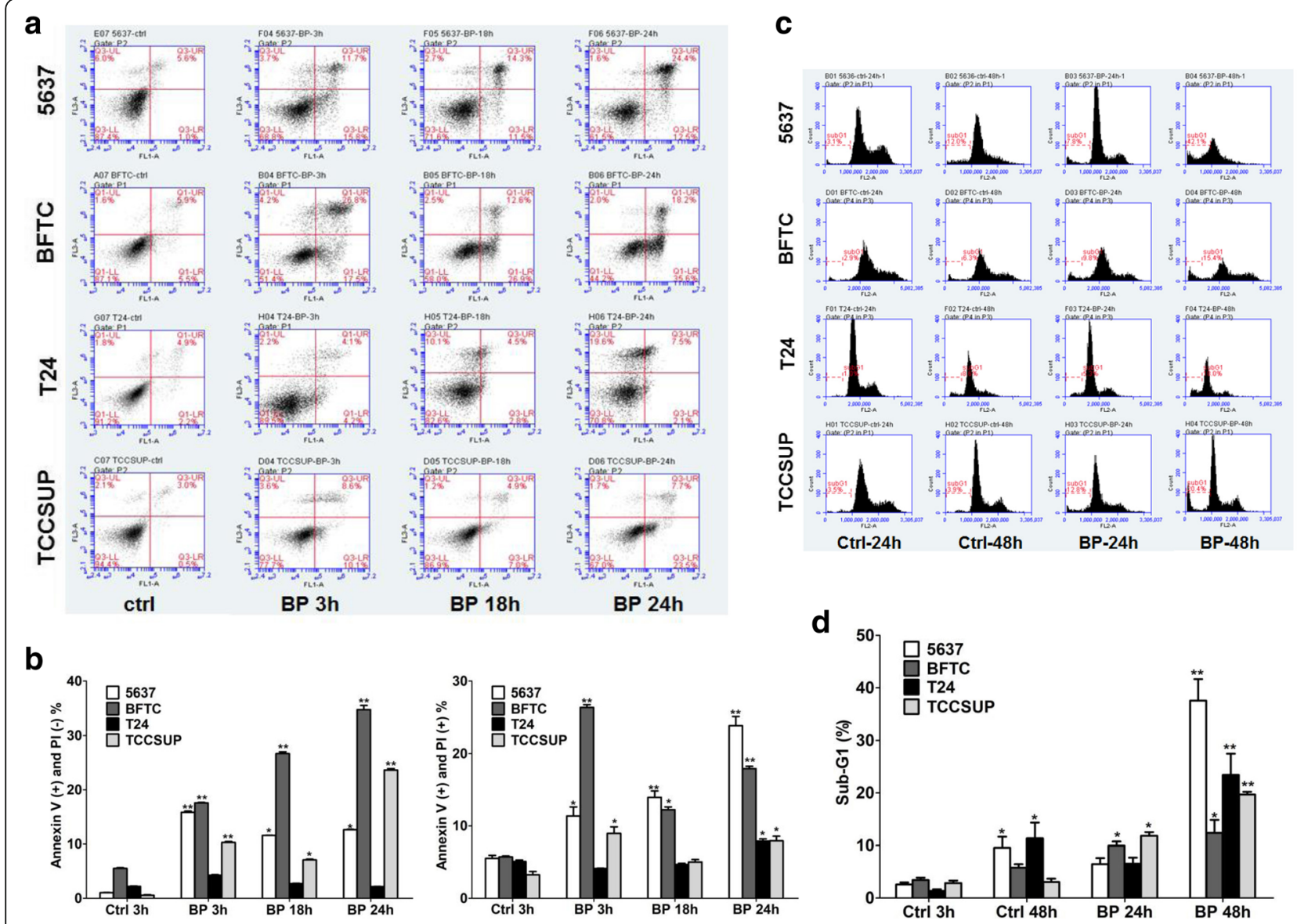

Fig. 2 Flow cytometric analysis of bladder cancer cells treated with BP. a Human bladder cancer cells were analyzed by annexin V-FITC staining in the vehicle control group (0.2\% DMSO) for $3 \mathrm{~h}$ or at presence of $60 \mu \mathrm{g} / \mathrm{ml}$ BP for 3,18 and $24 \mathrm{~h}$. $\mathbf{b}$ The percentage of annexin V-FITC positive population in bladder cancer cells in $\mathbf{a}$ is shown. $\mathbf{c}$ The accumulation of sub-G1 cell population in the presence or absence of $60 \mu \mathrm{g} / \mathrm{ml}$ BP for 24 and $48 \mathrm{~h}$. $\mathbf{d}$ The percentage of sub-G1 population in bladder cancer cells in $\mathbf{c}$ is shown

could affect the metastatic potential of bladder cancer cell lines, the expression levels of several EMT-related genes were examined using real-time RT-PCR. Upregulation of the mRNA expression of epithelial marker E-cadherin after treatment with BP for $24 \mathrm{~h}$ were 1.6 fold (5637), 1.35 fold (BFTC), 2.26 fold (T24), and 1.47 fold (TCCSUP), respectively. Down-regulation of the mRNA expression of mesenchymal marker $\mathrm{N}$-cadherin after treatment with BP for $24 \mathrm{~h}$ were 0.14 fold (5637), 0.7 fold (BFTC), 0.68 fold (T24), and 0.43 fold (TCCSUP), respectively (Fig. 5b). These results implicated that BP might inhibited the migration in bladder cancer cells via the modulation of EMT genes.

\section{Effect of BP on the combination therapy with cisplatin}

To investigate whether BP could enhance the therapeutic effects of chemotherapy agent such as cisplatin in combination therapy, the low dose of BP and cisplatin were used to evaluate their synergistic efficacy in bladder cancer cells. Human bladder cancer cells treated with cisplatin, or $\mathrm{BP}$, or in combination for $24 \mathrm{~h}$, were analyzed for viability with MTT assay. BP, at a lower dosage $(25 \mu \mathrm{g} / \mathrm{ml})$ showed synergistic cytotoxic effect when combined with cisplatin: 5637: 67.5\% (cisplatin alone: 90.5\%, BP alone: $83.1 \%$ ), BFTC: $90.1 \%$ (cisplatin alone: 99\%, BP alone: 99.3\%), T24: 59.8\% (cisplatin alone: 94.5\%, BP alone: $80.4 \%$ ) and TCCSUP: $62.4 \%$ (cisplatin alone: $90.1 \%$, BP alone: 92\%), respectively (Fig. 6). These data implicated the potential role of BP in combination therapy.

\section{BP suppressed tumor growth in NOD-SCID xenograft mice}

To evaluate the antitumor activity of BP in vivo, human bladder cancer xenograft were established by subcutaneous injection of $5 \times 10^{5}$ BFTC cells into the dorsal subcutaneous tissue of NOD-SCID mice. As shown in Fig. $7 \mathrm{a}$, the relative tumor volume in mice treated with 100 and $200 \mathrm{mg} / \mathrm{kg}$ BP was smaller than the vehicle treated control mice on day $26(44.2 \%, P=0.01 ; 35.9 \%$, 

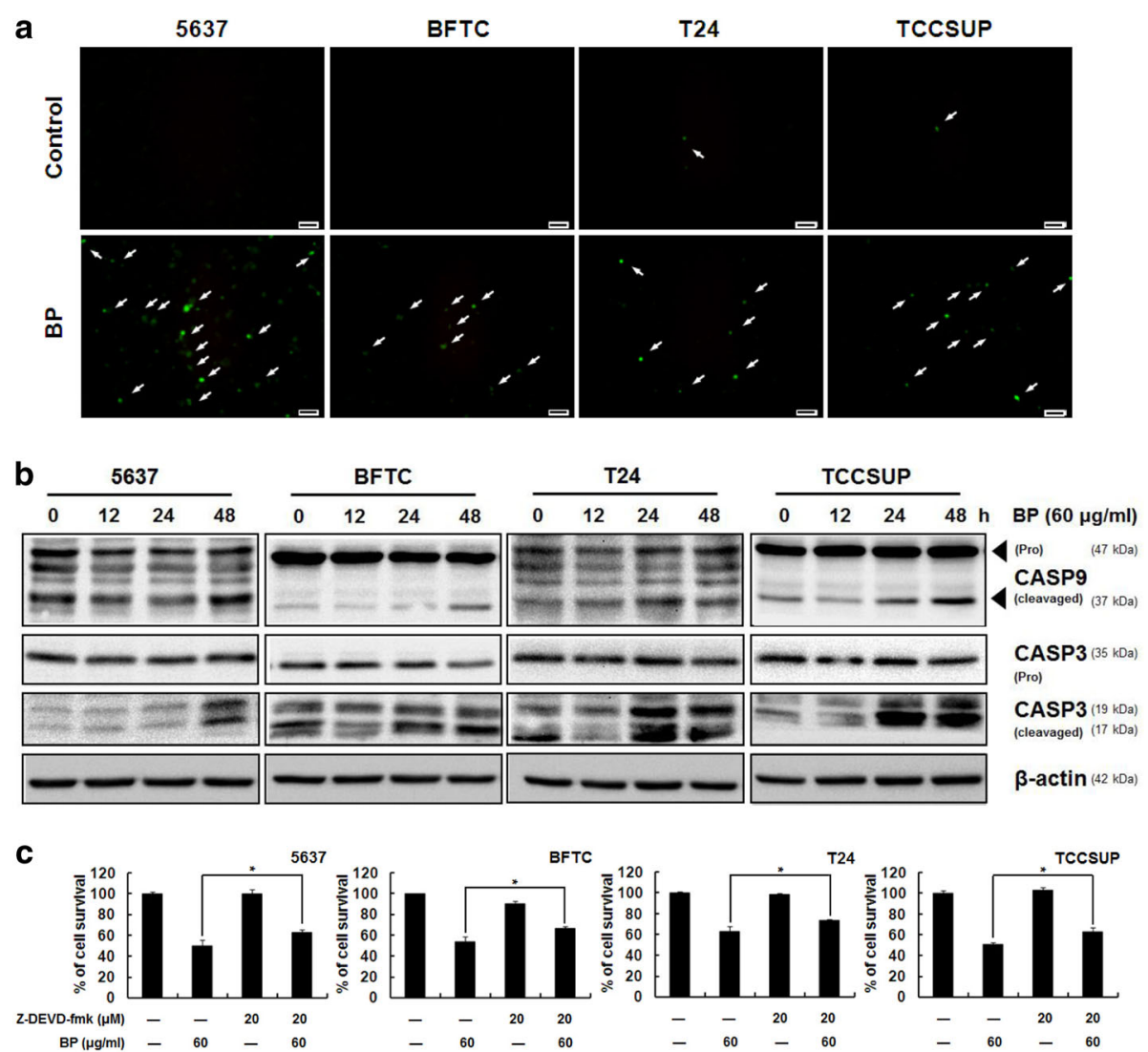

Fig. 3 BP induced mitochondrial-mediated apoptosis in human bladder cancer cells. a Human bladder cancer cells were treated with $0.2 \%$ DMSO (control) or $60 \mathrm{\mu g} / \mathrm{ml} \mathrm{BP}$ for $72 \mathrm{~h}$ and then stained with the TUNEL assay. TUNEL positive cells (green fluorescence) were indicated by arrows. Scale bar: $50 \mu \mathrm{m}$. b Human bladder cancer cells were treated with $60 \mu \mathrm{g} / \mathrm{ml}$ BP for 0 to $72 \mathrm{~h}$, and western blot analysis were performed for cleaved caspase-9 and -3. $\beta$-actin was used as an internal control. c MTT assay of human bladder cancer cells pretreated with caspase 3 inhibitor Z-DEVD-fmk (20 $\mu M$ ) for $1 \mathrm{~h}$ and then treated in the presence or absence of $60 \mu \mathrm{g} / \mathrm{ml} \mathrm{BP}$ for $48 \mathrm{~h}$. The values are the mean \pm S.D. from three independent experiments, ${ }^{*} p<$ 0.05 versus vehicle

$P=0.04$, respectively). Hematoxylin/eosin (H\&E) staining revealed that control group tumor specimens had large and irregular nuclei and exhibit squamous differentiation which are associated with a poor prognosis as compared to the BP-treated group (Fig. 7b). Furthermore, immunohistochemistry of the proliferation marker, Ki67, demonstrated that the control group showed increased Ki67 expression as compared to the BP-treated group (Fig. 7b). Caspase-3 activation was also observed in BP-treated tumors (Fig. 7c). There were no significant differences of body weight between the control and BP-treated groups.

\section{Discussion}

NHIRD is a population-based dataset which provides researchers to trace the medical service history covering over 99\% population in Taiwan, and is widely recognized to provide highly accurate diagnoses and clinical information [22, 23]. Several reports have used the NHIRD to evaluate the associations between different diseases [14,
19, 20]. As there were considerable differences in age, gender and comorbidities between the patients who has or has not been exposed to Angelica sinensis, propensity score matching with age and gender was applied to select the controls. The incidence of bladder cancer decreased in the patients who were exposure to Angelica sinensis using a matching method. However, the limitations of this study data from NHIRD should, however, be noted. One obvious limitation study is that, the incidence of bladder cancer was low and the numbers of bladder cancer were likely too small for these types of sub-analyses. The other limitations from NHIRD were described in previous studies $[19,20]$. Briefly, the severity of bladder cancer cannot be precisely extracted from ICD-9-CM codes and the database does not contain information on tobacco use, dietary habits, carcinogen exposure and body mass index, which may also be risk factors for bladder cancer were the limitations. In addition, the CHPs used might contain additional herbs other than Angelica Sinensis and additional bio-active 

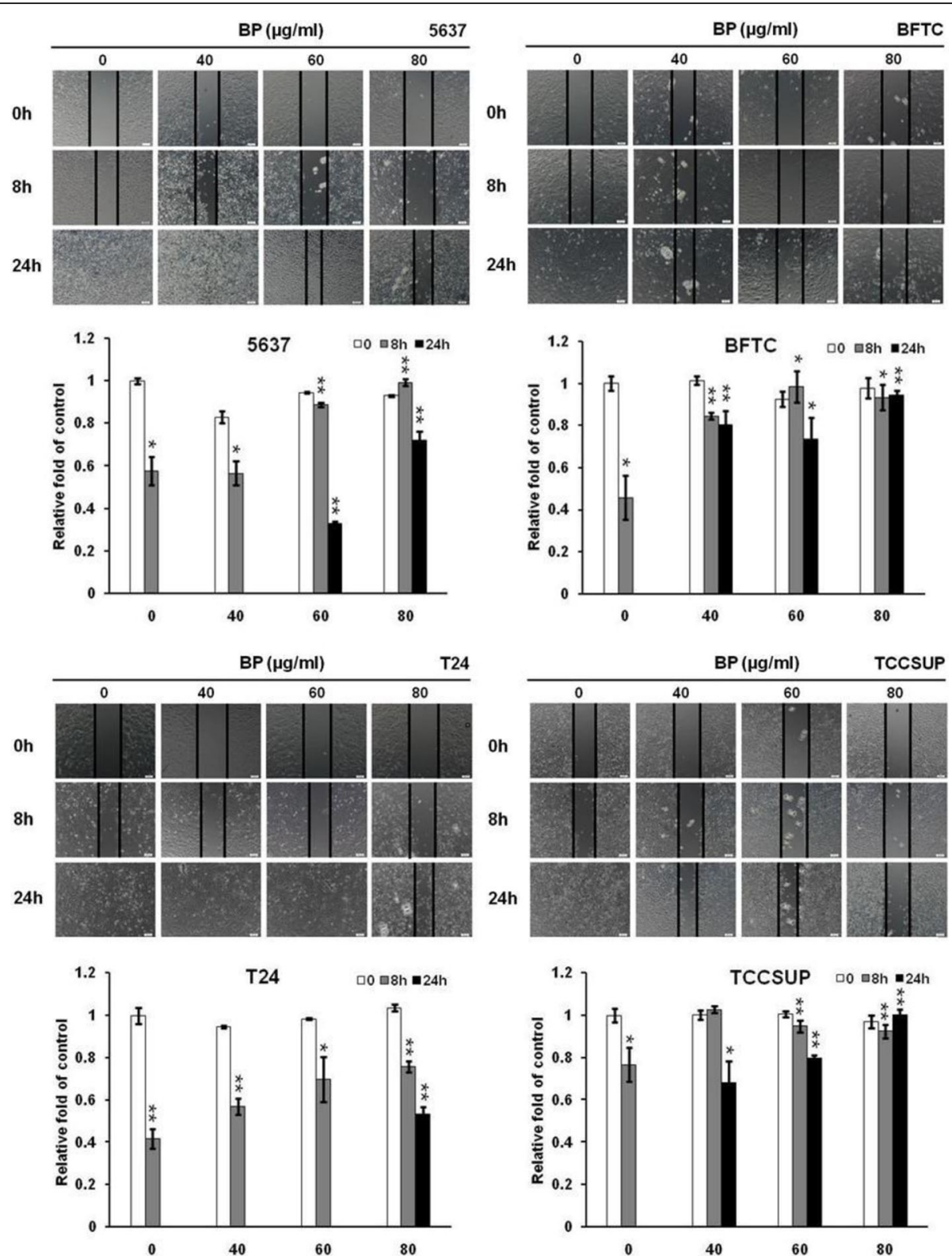

Fig. 4 The inhibitory effect of BP on the migration of human bladder cancer cells- wound healing assay. Human bladder cancer cells were treated with $0.2 \%$ DMSO as control or 40 to $80 \mu \mathrm{g} / \mathrm{ml}$ BP for the indicated time points. Images of wound closures were captured using inverted microscope with $100 \mathrm{X}$ magnification. The cell-free area invaded by migrated cells across the black lines were calculated by three randomized fields and quantified. The cell-free distance at $0 \mathrm{~h}$ were set at as 100\%. Data obtained from three independent experiments and presented as mean \pm S.D. from three independent experiments. Scale bar: $100 \mu \mathrm{m},{ }^{*} p<0.05$ versus vehicle; ${ }^{* *} p<0.01$ versus vehicle

agents. In order to clarify the results from the NHIRD study and the physiological effect of BP on bladder cancer cells, we thus carried out the in vitro and in vivo experiments.

Many dietary phytochemicals and extracts derived from herbs have been tested as antioxidants, or as inhibitors of cancer cell proliferation in vitro and in vivo [24]. Recent reports have demonstrated that BP is cytotoxic to various cancer cells including glioblastoma multiforme (GBM), hepatocellular carcinoma (HCC) and prostate cancer. However, the effect of BP on human bladder cancer cells has not been addressed. Based on the abovementioned discovery that patients who have been exposed to Angelica sinensis have lower incidence of bladder cancer, we thus intended to study the probable physiological effect of BP on bladder cancer using bladder cancer cell lines of various stages.

In the subsequent study, we demonstrated the cytotoxic effect of BP on various human bladder cancer cells. BP induced mitochondria-dependent apoptotic cell 


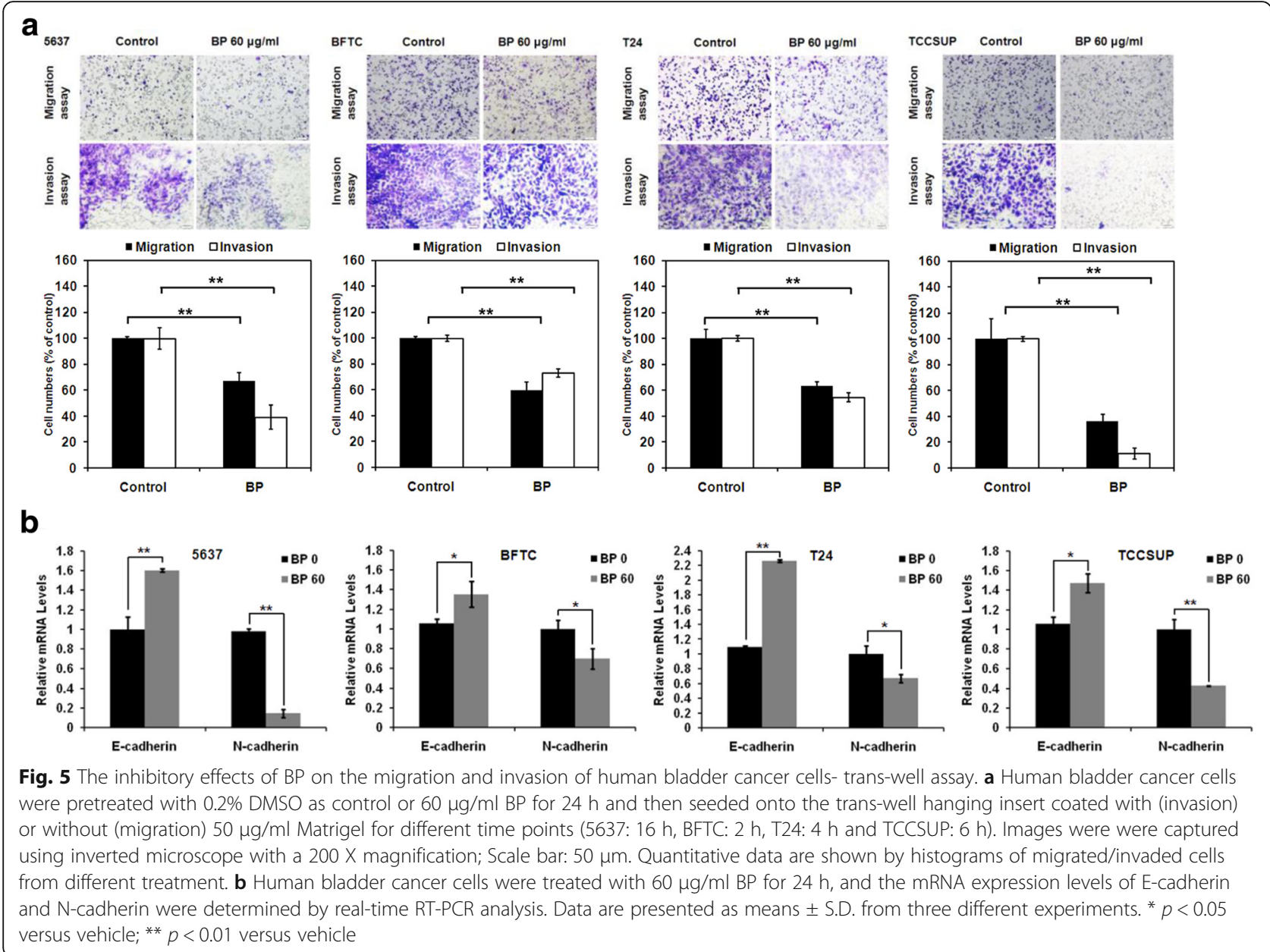

death, and inhibited the migration of the 4 bladder cancer cell lines. Interestingly, the 4 bladder cancer cell lines were isolated from different stages of bladder cancer. We thus speculated that BP might have therapeutic potential in treating bladder cancer of different stages. However, testing the therapeutic potential of BP in these stages of bladder cancers needs to be evaluated in different mouse models.

The mitochondria-dependent apoptotic cell death was evidenced by caspase- 9 and caspase- 3 cleavages in a time-dependent manner after BP treatments in these cell lines. Pretreatment of caspase-3 inhibitor (Z-DEVD-fmk) partly rescued the cells from BP cytotoxicity. The latestage apoptosis was revealed by the TUNEL assay. The ability of BP in eliciting apoptotic cell death had also been documented in brain, lung and prostate cancer cells in previous studies $[9-11,16]$.

Cell migration is essential for tumor metastasis, and metastasis is the most common fatal complication of all cancer patients [25]. The migration of human bladder cancer cell lines was suppressed by BP in this study, which implicated the anti-metastasis effect of $\mathrm{BP}$ on bladder cancer cells. Several crucial steps such as loss of cellular adhesion, increased the motility and invasiveness, has been found to be associated with epithelialmesenchymal transition (EMT) [26]. The up-regulation of N-cadherin promotes motility, invasiveness and metastasis in tumor cells, whereas the loss of E-cadherin reduces the E-cadherin-mediated cell-cell adhesion and progress toward malignancy. Thus, these events play critical roles in EMT [26]. Our results showed that BPpretreatment significantly inhibited the migration and invasion of bladder cancer cells (Fig. 5a). In addition, BP suppressed the expression of $\mathrm{N}$-cadherin but activated the expression of E-cadherin in the 4 bladder cancer cell lines (Fig. 5b). These results demonstrated the antimetastasis effect of BP on bladder cancer cells. We thus speculated that BP might have therapeutic potential in treatment of bladder cancer of all stages.

Synergistic analysis of different anticancer agents is an important approach to determine the ratio and/or dose of drugs for clinical combination application [27]. In previous studies, combination of cisplatin and other drugs could increase the cytotoxicity of chemotherapy in 


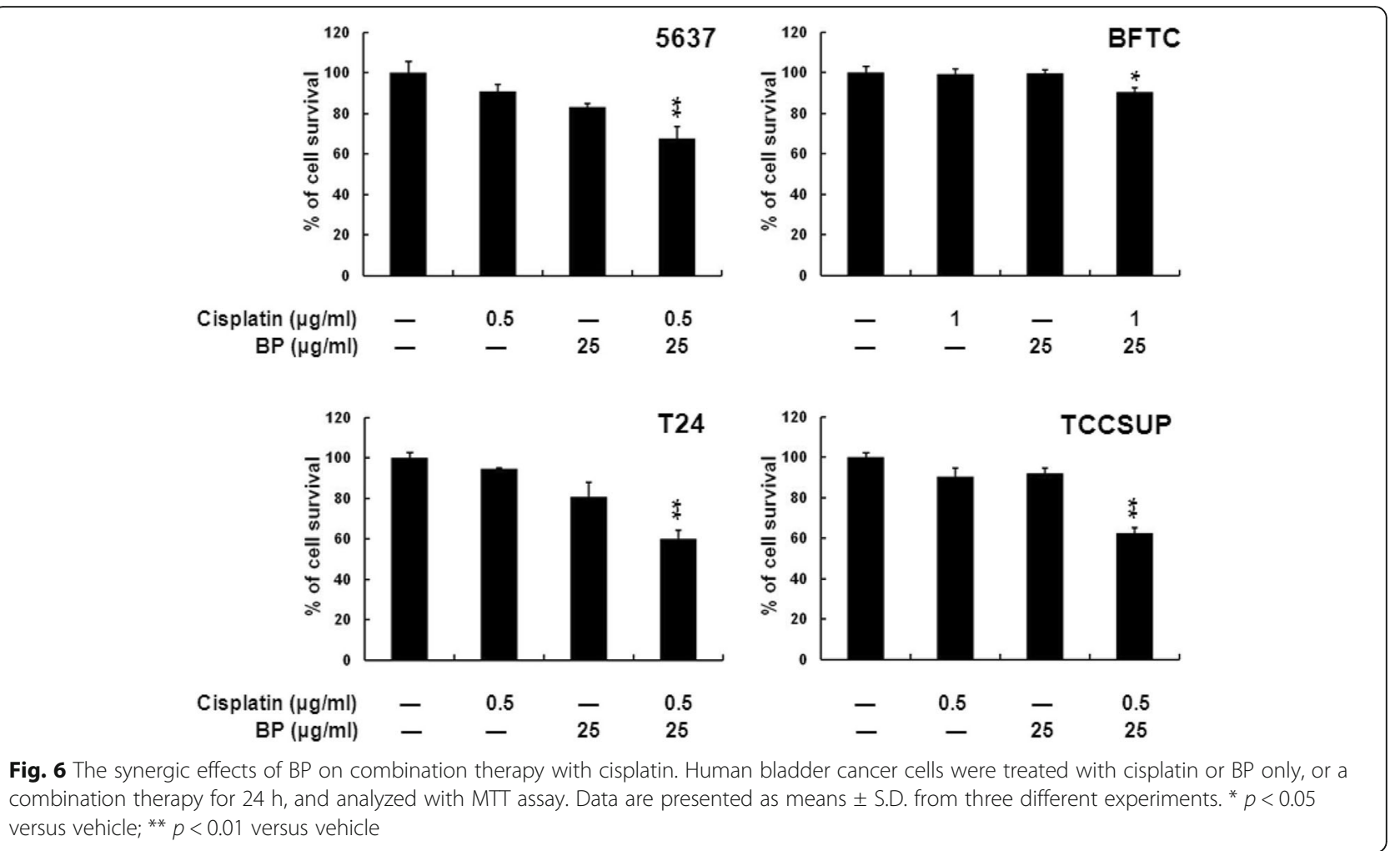

bladder cancer treatment $[28,29]$. In the present study, similar result of combined therapy was observed. Bladder cancer cells treated with BP demonstrated increased sensitivity to cisplatin, indicating that BP could be developed as a potential adjuvant cisplatin-based chemotherapy regimen. The mechanism of this synergistic effect includes drug inactivation, alterations in drug target, processing of drug-induced damage and evasion of apoptosis, which all needs to be clarified in the future [30].

\section{Conclusion}

In summary, the present study demonstrates the potential anti-proliferation and anti-metastatic activity of BP on human bladder cancer cells. BP exerted cytotoxicity by inducing apoptosis in human bladder cancer cells:

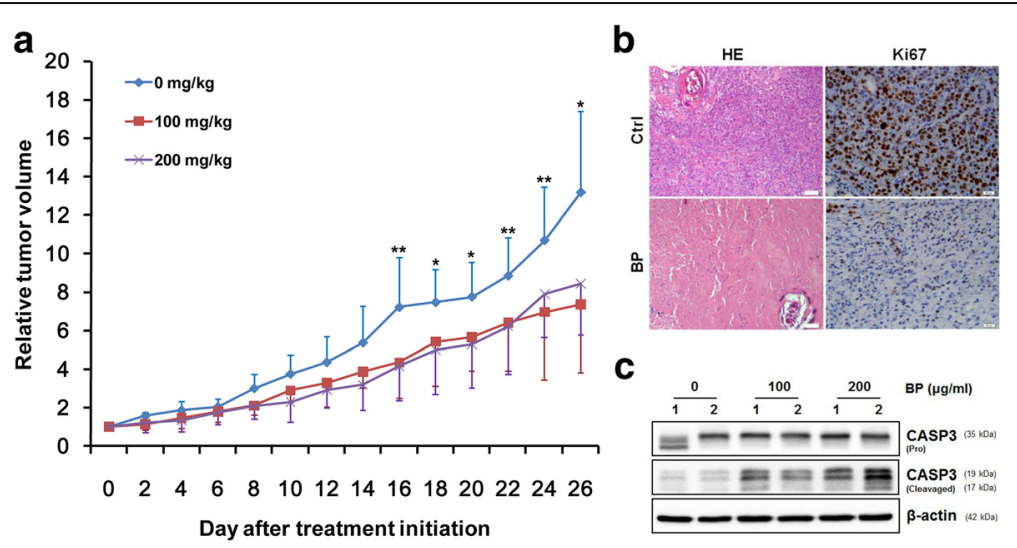

Fig. 7 BP inhibits xenographic growth of BFTC cells in NOD-SCID mice. a Approximately $5 \times 10^{5}$ BFTC cells were injected into the dorsal subcutaneous tissue of NOD-SCID mice. When the tumor reached $100-250 \mathrm{~mm}^{3}$, BFTC tumor-bearing mice were administrated s.c. with vehicle control or 100 to $200 \mathrm{mg} / \mathrm{kg}$ BP on days $0-4$ for 5 days. The relative tumor volumes of the control and the BP-treated groups are shown as means \pm S.D. of tumor volume at each time point. $\mathbf{b}$ Tumor tissue sections with HE staining. The proliferation marker ki67 were immuno-histochemically identified in the control and the BP-treated groups. Expression of cleavaged caspase-3 in the BFTC xenograft tumor tissue was up-regulated after BP administration as compared to control group by western blotting analysis c 
activations of caspases-9 and caspase- 3 were evidenced. The anti-metastatic effect of BP in human bladder cancer cells was shown by wound healing and trans-well assays. We have further demonstrated the tumor suppression potential in BFTC-xenograft animal models. In addition, the synergistic cytotoxic effect of BP in combination with cisplatin were also shown. An NHIRD database analysis showed lower incidence of bladder cancer in patients with exposure to Angelica sinensis, indicating the therapeutic potential of BP in clinical application.

\section{Additional file}

Additional file 1: Supplementary Methods. (DOC $48 \mathrm{~kb}$ )

\section{Abbreviations}

BP: n-butylidenephthalide; NHIRD: National Health Insurance Research Database

\section{Acknowledgements}

We thank to Ms. Hsin-Rong Wu, Department of Medical Research, Hualien Tzu Chi Hospital, for her assistance with animal experiments. This work was supported by grants from the Taichung Tzu Chi Hospital, Buddhist Tzu Chi Medical Foundation, Taichung, Taiwan (TTCRD104-10, TTCRD104-19,

TTCRD103-08, TTCRD103-12, TTCRD102-21).

\section{Funding}

This work was supported by grants from the Taichung Tzu Chi Hospital, Buddhist Tzu Chi Medical Foundation, Taichung, Taiwan (TTCRD104-10, TTCRD104-19, TTCRD103-08, TTCRD103-12, TTCRD102-21). The funders had no role in study design, data collection and analysis, decision to publish, or preparation of the manuscript.

\section{Availability of data and materials}

All data generated or analyzed during this study are included in this published article.

\section{Authors' contributions}

Conceived and designed the experiments: SCC, TLC, TFH, CYP; Performed the experiment: SCC, SYH, SFC, SPC; Contributed reagents/materials/analysis tools: TLC, TFH, SPC, CYP; Analyzed the data: SCC, SPC, SFC, SYH, TFH; Wrote the paper: SCC, TLC, SYH, SPC, CYP, TFH. All authors read and approved the final manuscript.

\section{Ethics approval and consent to participate}

Animal: The animal use protocol listed below has been reviewed and approved by Institutional Animal Care and Use Committee (IACUC), Hualien Tzu Chi Hospital, approval No: 104-13.

Human: The Institutional Review Board of Taichung Tzu Chi General Hospital in Taiwan approved the study protocol (REC103-43).

\section{Consent for publication}

Not applicable.

\section{Competing interests}

The authors declare that they have no competing interests.

\section{Publisher's Note}

Springer Nature remains neutral with regard to jurisdictional claims in published maps and institutional affiliations.

\section{Author details}

'Department of Research, Taichung Tzu Chi Hospital, Buddhist Tzu Chi Medical Foundation, Taichung, Taiwan. ²Department of Laboratory Medicine,
Taichung Tzu Chi Hospital, Buddhist Tzu Chi Medical Foundation, Taichung, Taiwan. ${ }^{3}$ General Education Center, Tzu Chi University of Science and Technology, Hualien, Taiwan. ${ }^{4}$ Division of Neuro-Oncology, Neuro-Medical Scientific Center, Hualien Tzu Chi Hospital, Buddhist Tzu Chi Medical Foundation, Hualien, Taiwan. ${ }^{5}$ Department of Ophthalmology, Mackay Memorial Hospital, Hsinchu, Taiwan. ${ }^{6}$ Tzu Chi Stem Cells Center, Hualien Tzu Chi Hospital, Buddhist Tzu Chi Medical Foundation, Hualien, Taiwan. ${ }^{7}$ Department of Medical Research, Hualien Tzu Chi Hospital, Buddhist Tzu Chi Medical Foundation, Hualien, Taiwan. ${ }^{8}$ Institute of Medical Sciences, School of Medicine, Tzu Chi University, 701, Section 3, Chung-Yang Road, Hualien 970, Taiwan. ${ }^{9}$ Department of Urology, Taichung Tzu Chi Hospital, Buddhist Tzu Chi Medical Foundation, Section 1, Fengxing Road, Tanzi Dist., Taichung City 427, Taiwan. ${ }^{10}$ School of Medicine, Tzu Chi University, Hualien, Taiwan.

Received: 16 February 2017 Accepted: 28 November 2017

Published online: 06 December 2017

\section{References}

1. Siegel R, Ward E, Brawley O, Jemal A. Cancer statistics, 2011: the impact of eliminating socioeconomic and racial disparities on premature cancer deaths. CA Cancer J Clin. 2011;61(4):212-36.

2. Siegel RL, Miller KD, Jemal A. Cancer statistics, 2015. CA Cancer J Clin. 2015; 65(1):5-29.

3. Lutzeyer $\mathrm{W}$, Rubben $\mathrm{H}$, Dahm H. Prognostic parameters in superficial bladder cancer: an analysis of 315 cases. J Urol. 1982;127(2):250-2.

4. Audenet F, Yates DR, Cussenot $O$, Roupret $M$. The role of chemotherapy in the treatment of urothelial cell carcinoma of the upper urinary tract (UUT-UCC). Urol Oncol. 2013;31(4):407-13.

5. Calabro F, Sternberg CN. Neoadjuvant and adjuvant chemotherapy in muscle-invasive bladder cancer. Eur Urol. 2009;55(2):348-58.

6. Leow JJ, Martin-Doyle W, Rajagopal PS, Patel CG, Anderson EM, Rothman AT, Cote RJ, Urun Y, Chang SL, Choueiri TK, et al. Adjuvant chemotherapy for invasive bladder cancer: a 2013 updated systematic review and meta-analysis of randomized trials. Eur Urol. 2014;66(1):42-54.

7. XR W. Urothelial tumorigenesis: a tale of divergent pathways. Nat Rev Cancer. 2005;5(9):713-25.

8. Chen YL, Jian MH, Lin CC, Kang JC, Chen SP, Lin PC, Hung PJ, Chen JR, Chang WL, Lin SZ, et al. The induction of orphan nuclear receptor Nur77 expression by $\mathrm{n}$-butylenephthalide as pharmaceuticals on hepatocellular carcinoma cell therapy. Mol Pharmacol. 2008;74(4):1046-58.

9. Chiu SC, Chen SP, Huang SY, Wang MJ, Lin SZ, Harn HJ, Pang CY. Induction of apoptosis coupled to endoplasmic reticulum stress in human prostate cancer cells by n-butylidenephthalide. PLoS One. 2012;7(3):e33742.

10. Lin PC, Chen YL, Chiu SC, YL Y, Chen SP, Chien MH, Chen KY, Chang WL, Lin SZ, Chiou TW, et al. Orphan nuclear receptor, Nurr-77 was a possible target gene of butylidenephthalide chemotherapy on glioblastoma multiform brain tumor. J Neurochem. 2008;106(3):1017-26.

11. Liu PY, Sheu JJ, Lin PC, Lin CT, Liu YJ, Ho LI, Chang LF, WC W, Chen SR, Chen J, et al. Expression of Nur77 induced by an n-butylidenephthalide derivative promotes apoptosis and inhibits cell growth in oral squamous cell carcinoma. Invest New Drugs. 2012;30(1):79-89.

12. Tsai NM, Chen YL, Lee CC, Lin PC, Cheng YL, Chang WL, Lin SZ, Harn HJ. The natural compound n-butylidenephthalide derived from Angelica Sinensis inhibits malignant brain tumor growth in vitro and in vivo. J Neurochem. 2006;99(4):1251-62.

13. Wei CW, Lin CC, YL Y, Lin CY, Lin PC, MT W, Chen CJ, Chang W, Lin SZ, Chen $Y L$, et al. N-Butylidenephthalide induced apoptosis in the A549 human lung adenocarcinoma cell line by coupled down-regulation of AP-2alpha and telomerase activity. Acta Pharmacol Sin. 2009;30(9):1297-306.

14. Lin CC, Chiang JH, Li Cl, Liu CS, Lin WY, Hsieh TF, Li TC. Cancer risks among patients with type 2 diabetes: a 10-year follow-up study of a nationwide population-based cohort in Taiwan. BMC Cancer. 2014;14:381.

15. CTW, Lai JN, Tsai YT. The prescription pattern of Chinese herbal products that contain dang-qui and risk of endometrial cancer among tamoxifen-treated female breast cancer survivors in Taiwan: a population-based study. PLoS One. 2014;9(12):e113887.

16. Chiang TL. Taiwan's 1995 health care reform. Health Policy. 1997;39(3): 225-39.

17. Tseng $\mathrm{CH}$. Mortality and causes of death in a national sample of diabetic patients in Taiwan. Diabetes Care. 2004;27(7):1605-9. 
18. Charlson ME, Pompei P, Ales KL, MacKenzie CR. A new method of classifying prognostic comorbidity in longitudinal studies: development and validation. J Chronic Dis. 1987;40(5):373-83.

19. Hsieh TF, Yang YW, Lee SS, Lin TH, Liu HH, Tsai TH, Chen CC, Huang YS, Lee CC. Use of 5-alpha-reductase inhibitors did not increase the risk of cardiovascular diseases in patients with benign prostate hyperplasia: a fiveyear follow-up study. PLoS One. 2015;10(3):e0119694.

20. Yang YW, Hsieh TF, CH Y, Huang YS, Lee CC, Tsai TH. Zolpidem and the risk of Parkinson's disease: a nationwide population-based study. J Psychiatr Res. 2014;58:84-8.

21. Lin HC, Chao PZ, Lee HC. Sudden sensorineural hearing loss increases the risk of stroke: a 5-year follow-up study. Stroke. 2008;39(10):2744-8.

22. Cheng $\mathrm{CL}$, Kao YH, Lin SJ, Lee CH, Lai ML. Validation of the National Health Insurance Research Database with ischemic stroke cases in Taiwan. Pharmacoepidemiol Drug Saf. 2011;20(3):236-42.

23. Cheng $C L$, Lee CH, Chen PS, Li YH, Lin SJ, Yang YH. Validation of acute myocardial infarction cases in the national health insurance research database in taiwan. J Epidemiol. 2014;24(6):500-7.

24. Shu L, Cheung KL, Khor TO, Chen C, Kong AN. Phytochemicals: cancer chemoprevention and suppression of tumor onset and metastasis. Cancer Metastasis Rev. 2010;29(3):483-502.

25. Yamaguchi $\mathrm{H}$, Wyckoff J, Condeelis J. Cell migration in tumors. Curr Opin Cell Biol. 2005;17(5):559-64.

26. Gupta GP, Massague J. Cancer Metastasis: building a framework. Cell. 2006; 127(4):679-95.

27. Lu Y, Li CS, Dong Q. Chinese herb related molecules of cancer-cellapoptosis: a minireview of progress between Kanglaite injection and related genes. J Exp Clin Cancer Res. 2008;27:31.

28. Chiu SC, Huang SY, Chang SF, Chen SP, Chen CC, Lin TH, Liu HH, Tsai TH, Lee SS, Pang CY, et al. Potential therapeutic roles of tanshinone IIA in human bladder cancer cells. Int J Mol Sci. 2014;15(9):15622-37.

29. Hsieh TF, Chen CC, Ma WL, Chuang WM, Hung XF, Tsai YR, Lin MH, Zhang Q, Zhang C, Chang C, et al. Epidermal growth factor enhances androgen receptormediated bladder cancer progression and invasion via potentiation of AR transactivation. Oncol Rep. 2013;30(6):2917-22.

30. Longley DB, Johnston PG. Molecular mechanisms of drug resistance. J Pathol. 2005:205(2):275-92.

\section{Submit your next manuscript to BioMed Central and we will help you at every step:}

- We accept pre-submission inquiries

- Our selector tool helps you to find the most relevant journal

- We provide round the clock customer support

- Convenient online submission

- Thorough peer review

- Inclusion in PubMed and all major indexing services

- Maximum visibility for your research

Submit your manuscript at www.biomedcentral.com/submit

) Biomed Central 